\title{
Association of post-stroke disability with plasma concentration and anti-Xa activity of apixaban.

Masaya Kumamoto MD, ${ }^{1}$ Kazunori Toyoda MD, ${ }^{1}$ Shinichi Wada MD, ${ }^{1}$ Naoki Tagawa MD, ${ }^{1}$ Takayuki Matsuki MD, ${ }^{1}$ Takuya Okata MD, ${ }^{1}$ Akira Okamoto MT2 , Toshiyuki Miyata PhD, ${ }^{2}$ Masafumi Ihara MD, ${ }^{3}$ Masatoshi Koga MD ${ }^{1}$

${ }^{1}$ Department of Cerebrovascular Medicine, ${ }^{2}$ Divsion of Clinical Chemistry, ${ }^{3}$ Department of Neurology, National Cerebral and Cardiovascular Center, Suita, Osaka, Japan

\section{Background \& Objectives}

$\checkmark$ Several studies suggested that physical vulnerability, so-called frailty, affects pharmacokinetics ${ }^{1}$.

$\checkmark$ Patients in acute stroke also show physical vulnerability ${ }^{2}$, but its relation with pharmacokinetics of anticoaglants remains unknown.

$\checkmark$ This study aimed to determine whether post-stroke disability was associated with pharmacokinetics of apixaban in acute ischemic stroke patients.

\section{Methods}

$\checkmark$ This single-center, prospective observational study included patients with acute ischemic stroke or transient ischemic attack (TIA) who were admitted to our hospital within 7 days after onset and started apixaban for non-valvular atrial fibrillation from JAN 2012 to DEC 2015.

$\checkmark$ Patients were divided into two groups according to presence of mild (mRS 0-3) and severe disability (mRS 4-5) at discharge.

$\checkmark$ Blood sample was obtained before and 4 hours after taking apixaban. Anti-Xa activity (AXA) was measured using chromogenic assay, (STA ${ }^{\circledR}$-Liquid Anti-Xa).

Concentration (C) of apixaban was analyzed based on AXA.

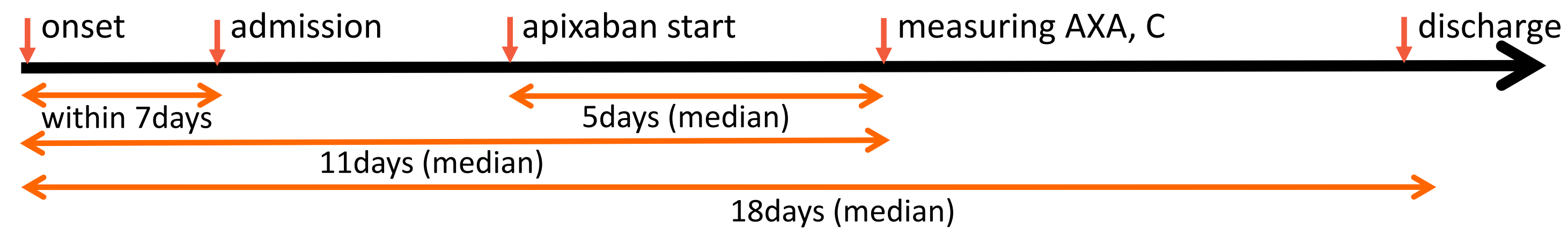

\section{Results}

Baseline characteristics

\begin{tabular}{|c|c|c|c|c|}
\hline & Overall & $\begin{array}{c}\text { mild } \\
\text { disability }\end{array}$ & $\begin{array}{c}\text { severe } \\
\text { disability }\end{array}$ & P- Value \\
\hline & $n=167$ & $n=123$ & $n=44$ & \\
\hline Women & $70(41.9)$ & $52(42.3)$ & $18(40.9)$ & 0.875 \\
\hline Age, y & $78.8 \pm 9$ & $79.1 \pm 9$ & $78.1 \pm 9$ & 0.552 \\
\hline Body weight, kg & $55.1 \pm 12.3$ & $54.3 \pm 11.6$ & $57.2 \pm 14.1$ & 0.179 \\
\hline Serrum creatinin, $\mathrm{mg} / \mathrm{dl}$ & $0.91 \pm 0.2$ & $0.91 \pm 0.3$ & $0.92 \pm 0.2$ & 0.932 \\
\hline Creatinine clearance, $\mathrm{ml} / \mathrm{min}$ & $52.9 \pm 24.2$ & $52.2 \pm 23.3$ & $54.9 \pm 26.9$ & 0.524 \\
\hline Prestroke $\mathrm{CHADS}_{2}$ score & $2[2-3]$ & $2[2-4]$ & $2[2-3]$ & 0.576 \\
\hline restroke $\mathrm{CHA}_{2} \mathrm{DS}_{2}$-VASc score & $4[3-5]$ & $4[3-5]$ & $4[3-6]$ & 0.972 \\
\hline Prestroke HAS-BLED score & $3[2-3]$ & $3[2-3]$ & $2[2-4]$ & 0.893 \\
\hline Prestroke mRS & $0[0-0]$ & $0[0-0]$ & $0[0-0]$ & 0.114 \\
\hline NIHSS on admission & $6[2-12]$ & $4[2-12]$ & 15 [9-19] & $<0.001$ \\
\hline Regular dosage of apixaban & $89(53.3)$ & $64(52.0)$ & $25(56.8)$ & 0.585 \\
\hline rom onset to measuring apixaban & $11[8-16]$ & $10[7-13]$ & $15[11-22]$ & 0.509 \\
\hline
\end{tabular}

Multiple regression analysis for variables predicting AXA/C
Relationship between $\mathrm{AXA} / \mathrm{C}$ of apixaban and disability

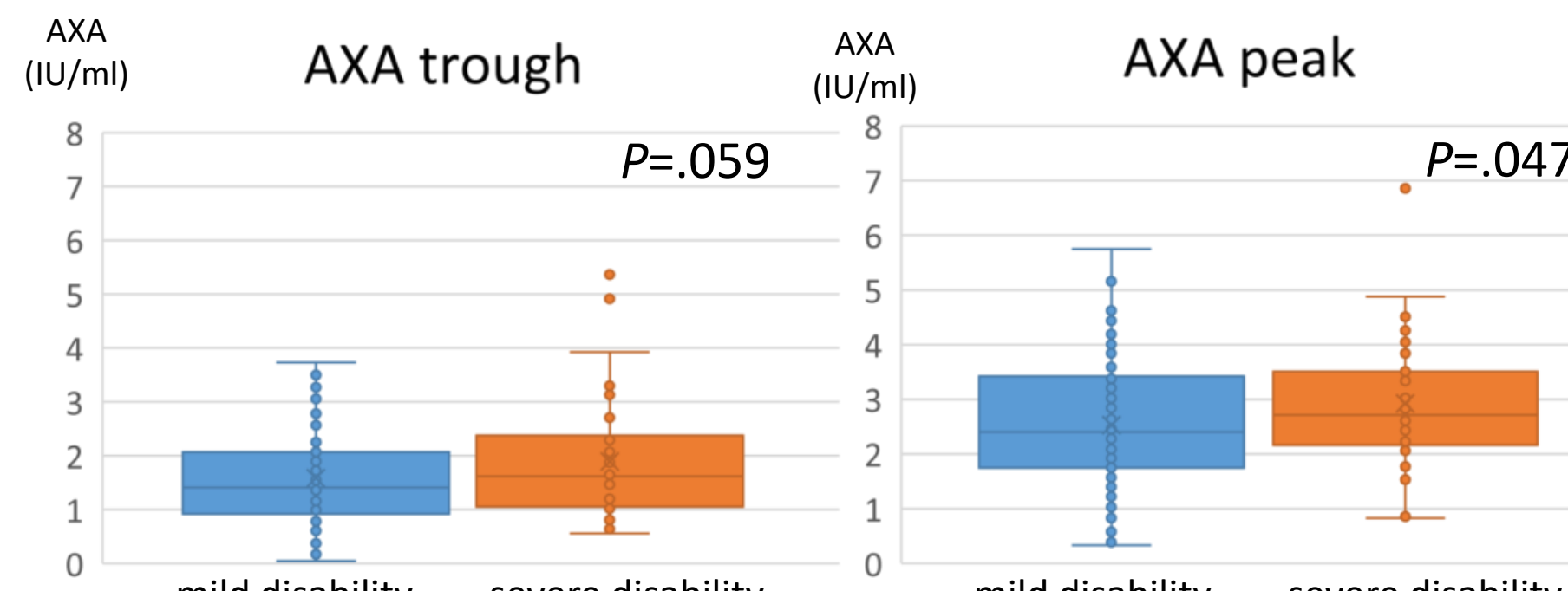

mild disability severe disability $2.53(2.33-2.73) \quad 2.92(2.59-3.26)$

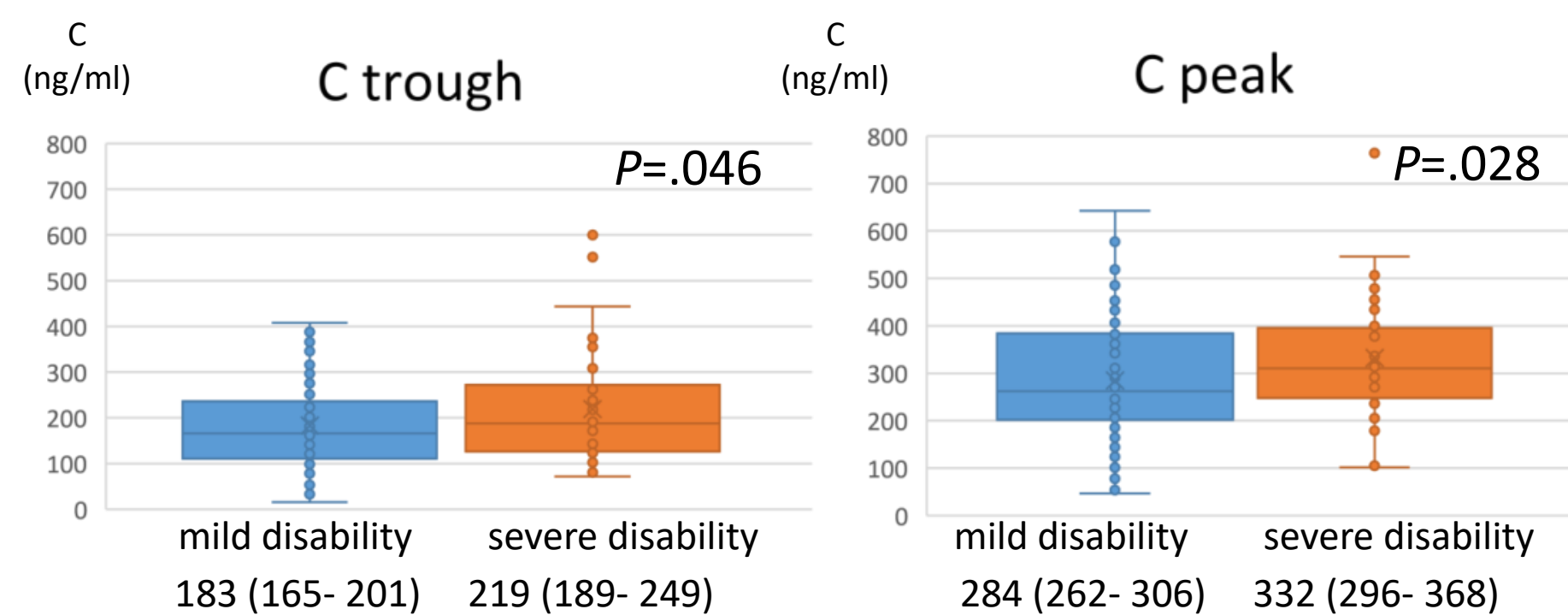

\begin{tabular}{|c|c|c|c|c|c|c|c|c|c|c|c|c|}
\hline \multirow[b]{2}{*}{ Variables } & \multicolumn{3}{|c|}{ AXA trough } & \multicolumn{3}{|c|}{ AXA peak } & \multicolumn{3}{|c|}{ C trough } & \multicolumn{3}{|c|}{ C peak } \\
\hline & $B$ & SE B & $\beta$ & $\mathrm{B}$ & SE B & $\beta$ & $B$ & SE B & $\beta$ & $\mathrm{B}$ & SE B & $\beta$ \\
\hline$\overline{\text { Age, } y}$ & 0.03 & 0.01 & $.30^{* *}$ & 0.03 & 0.01 & $.22 * *$ & 3.33 & 1.0 & $.30^{* *}$ & 3.31 & 1.2 & $.24 * *$ \\
\hline Body weight, kg & -0.01 & 0.01 & $-.19 *$ & -0.02 & 0.01 & $-.21 *$ & -1.47 & 0.8 & $-.18^{*}$ & -1.96 & 0.9 & $-.20 *$ \\
\hline Serum creatinine, $\mathrm{mg} / \mathrm{dl}$ & 0.88 & 0.24 & $.27 *$ & 0.85 & 0.30 & $.21^{*}$ & 90.5 & 26.7 & $.25 *$ & 68.35 & 32 & $.16^{*}$ \\
\hline Regular dosage of apixaban & 1.01 & 0.19 & $.54^{* *}$ & 1.40 & 0.23 & $.62^{* *}$ & 107.7 & 20.7 & $.53^{* *}$ & 155.62 & 24.8 & $.63^{* *}$ \\
\hline Severe disability & 0.32 & 0.15 & $.16^{*}$ & 0.41 & 0.18 & $.16^{*}$ & 36.6 & 16.1 & $.16^{*}$ & 47.63 & 19.3 & $.17^{*}$ \\
\hline$R^{2}$ & \multicolumn{3}{|c|}{0.22} & \multicolumn{3}{|c|}{0.24} & \multicolumn{3}{|c|}{0.21} & \multicolumn{3}{|c|}{0.24} \\
\hline$F$ for change in $R^{2}$ & \multicolumn{3}{|c|}{$9.2^{* *}$} & \multicolumn{3}{|c|}{$10.3 * *$} & \multicolumn{3}{|c|}{$8.5^{* *}$} & \multicolumn{3}{|c|}{$10.1 * *$} \\
\hline
\end{tabular}

${ }^{*} \mathrm{p}<.05,{ }^{* *} \mathrm{p}<.01$

\section{Conclusions}

Post-stroke disability was independently associated with pharmacokinetics of apixaban.

\section{References}

1. Hilmer SN : Br J Clin Pharmacol. 2011;71(2):224-31.

2. Scherbakov N: Stroke. 2011;42:3646-3650.

3. Dávalos A: Stroke. 1996;27:1028-1032

\section{Disclosure}

Cardiovascular Diseases of the National Cerebral and Cardiovascular Center, and Grants from the Japan Agency for Medical Research and Development (AMED: 17ek0210091h0001, 17ek0210055h0001). 